\title{
Article \\ Theoretical investigation of glycine micro-solvated. Energy and NMR spin spin coupling constants calculations
}

\author{
M. Cristina Caputo ${ }^{1}$ and Patricio F. Provasi ${ }^{2}$ (D)000-0002-9773-5542 \\ 1 Departamento de Física - IFIBA - Universidad de Buenos Aires, Buenos Aires, Argentina; \\ caputo@df.uba.ar \\ 2 Department of Physics - IMIT, University of Northeastern, Av. Libertad 5500, Corrientes, \\ Argentina; patricio@unne.edu.ar \\ * Correspondence: patricio@unne.edu.ar
}

\begin{abstract}
Glycine in its neutral form can exist in the gas phase while its zwitterion form is more stable in water solution.But how many waters are actually necessary to stabilize the zwitterionic structure in the gas phase? Are the intramolecular isotropic spin spin coupling constants sensitive enough to accuse the change in the environment? or the conformer observed? These and related questions have been investigated by a computational study at the level of density functional theory employing the B3LYP functional and the $6-31++\mathrm{G}^{* *}$-J basis set.We found that at least two water molecules explicitly accounted in the super-molecule structure are necessary to stabilize both conformers of glycine within a water polarizable continuum model. At least half of the SSCC's of both conformers are very stable to changes in the environment and at least four of them differ significantly between Neutral and Zwitterion conformation.
\end{abstract}

Keywords: Glycine; Microsolvation; Energy; NMR; SSCC

\section{Introduction}

Glycine is the smallest amino-acid conforming living organisms, and therefore of great interest in theoretical studies mainly due to the great relative computational saving its investigation demand. In particular, many of its properties are already known experimentally [1-9]. Among the diversity of works on glycine, some are aimed to elucidate the mechanisms of tautomerization. Hence the proton transfer of the neutral glycine, stable in the gas phase, to zwitterionic conformer, stable in aqueous solution, has an estimated free energy barrier of about $7.3 \frac{\mathrm{kcal}}{\mathrm{mol}}[1-3,5,6,8,10-14]$. Some other recent works are aimed to study small clusters of Glycine: $\left(\mathrm{H}_{2} \mathrm{O}\right)_{n}$ complexes in order to elucidate structures and binding energies [15-18], or the stabilization of Z-Glycine in water and its NMR signature [19] and the election of a method for fast accurate of ${ }^{1} J(C, H)$ for application in proteins [20]

Császár [21] found the three most stable conformers of neutral glycine and named them Ip, IIp and IIIp), then Godfrey et al [22] found a slight different set and named them 1, 3 and 2, later Sauer et al [23] and Aikens and Gordon [4] made the same finding as Császár's naming the conformers respectively $A, C$ and $B$ and N1, N3 and N2). More recently Caputo et al [24] found the structures proposed by Császár.

According to Császár [21] and Ding and Krogh-Jespersen [25] the zwitterion spontaneously tend to structure IIp, 2, C or N3 when it is let in gas phase which is obviously the shorter path for the proton. Moreover, the most stable geometry we found when including a continuum polarizable medium $(\mathrm{PCM})$ is precisely this last one, named in the present work by $\mathrm{N}$ for neutral. This conformer corresponds to the second most stable structure found by Császár, Sauer et al, Aikens and Gordon and Caputo et al. Correspondingly, we name the other two structures analyzed in this work with Z (for zwitterion) and TS (for transition state) which were found within the PCM model. 
Alonso et. al [16] reported that the conformer of glycine III, which coincide with the neutral conformer chosen for this work, in the complex glycine:water IIIa was not observed in their experiment based in a supersonic jet and they explain the reason as due to its collisional relaxation to the most stable conformer of glycine (I). However it is not the purpose of the present work to analyze the collisions glycine:water.

In this work we use structure IIp, 2, C or N3 for the neutral glycine and its zwitterion to analyze their stability within the PCM model and including explicit water molecules, up to reaching saturation of the hydrophilic positions.

The number of water molecules necessary to reach saturation vary a bit according to the conditions established for the hydrogen bond (HB) formation, see for instance Ref. [6]. Hence, we only restricted rings of water molecules that do not involve the glycine in them, so the saturation is reached with eight molecules of water. The energies scheme arising from it allows one to analyze the contributions in energy of the HB's formed in the super-molecule model. The scheme contemplated for adding water molecules is shown in the computational details section. Finally we analyze the NMR indirect spin-spin coupling constants $(J)$ between the intramolecular heavy atoms.

\section{Materials and Methods}

The calculations were performed with Gussian 09 suit of program [26]. The geometry optimization were done at B3LYP [27,28] with 6-31+G(d,p) [29-32] basis sets. The local minima have passed the frequency test.

The calculations of SSCC's were done using the density functional theory with the B3LYP functional, and employed a reliable basis set $6-311++\mathrm{G}^{* *} \mathrm{~J}$ [33]. Basis functions, for both glycine and water molecule, were taken from Basis Set Exchange [34-36].

Calculations include a liquid solvent described by Polarizable Continuum Model (PCM) approximation [37] with the variant (SMD) [38] that differs from the default (IEF-PCM) also by Truhlar and co-workers solvation model [39-43] that do not include the electrostatic terms. Vibrational corrections were not included due to these are normally too expensive to calculate in standard applications to larger molecules.

The geometries obtained in this work are essentially the same as the ones obtained by Caputo et al in Ref. [24] and are represented in Figure 1, where the transition state (TS) was found with the help of $\mathrm{N}$ and Z structures within the PCM approximation mentioned above and QST3 option.

Subsequently water molecules were added following the order and position indicated in Figure 2 and Table 1. Up to eight water molecules effectively joined to glycine were feasible to be included for the three conformers, $i$. $e$. without having rings of water molecules which do not include the glycine in them.
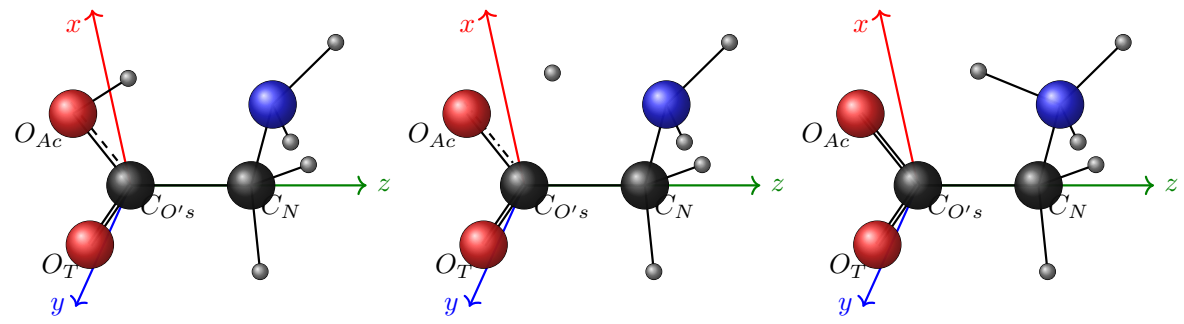

Figure 1. Optimized geometries of glycine neutral form: N (left) Neutral, TS (centre) Transition State and Z (right) Zwitterionic form: using B3LYP/6-31+G(d,p)

The theory of indirect nuclear SSCC's, [44] and different computational methods for calculating them has been described extensively in the literature [45-51]. However, it should be useful, as a reminder, to mention that there are four contributions to the SSCC: the Fermi contact (FC) and the spin-dipolar (SD), which come from the interaction of the nuclear magnetic moments with the spin of the electrons, as well as the diamagnetic spin 
Table 1. The possible H-bond sites between $\mathrm{H}_{2} \mathrm{O}$ and N-Gly/Z-Gly

\begin{tabular}{c|l|l}
\hline H-bond sites & $\mathrm{N} / \mathrm{Z}-\mathrm{Structure}$ & insertion order \\
\hline $\mathrm{Ac}$ & $\mathrm{H}-\mathrm{O}-\mathrm{H} \cdots(\mathrm{OH})-\mathrm{C}$ & 1,6 \\
$\mathrm{Am}$ & $\mathrm{H}_{2} \mathrm{O} \cdots \mathrm{H}_{2} \mathrm{~N}-\mathrm{C}$ & 2,4 \\
$\mathrm{O}_{T}$ & $\mathrm{H}-\mathrm{O}-\mathrm{H} \cdots \mathrm{O}=\mathrm{C}$ & $3,5,7$ \\
$\mathrm{AmAc}$ & $\mathrm{C}-\mathrm{O}-\mathrm{H} \cdots(\mathrm{OH})-\mathrm{H} \cdots\left(\mathrm{NH}_{2}\right)-\mathrm{C}$ & 8 \\
\hline
\end{tabular}
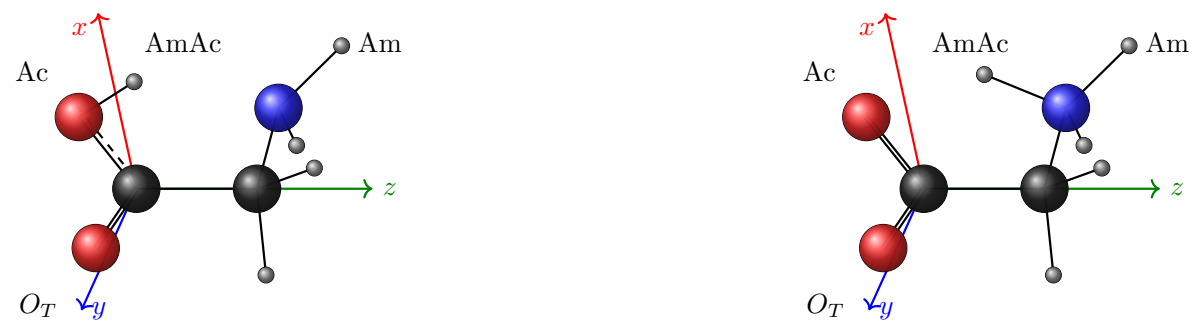

Figure 2. The possible $\mathrm{H}$-bonds between $\mathrm{H}_{2} \mathrm{O}$ and N-Gly (left) / Z-Gly (right)

orbital (DSO) and the paramagnetic spin orbital (PSO), which are due to the interaction of the nuclear spins with the orbital angular momentum of the electrons.

\section{Results and Discussion}

\subsection{Basics Concepts}

We have analyzed the hydration of one conformer of glycine, stable in gas phase, its zwitterion counterpart, which is stable in liquid phase, and the transition state obtained from the former two, depicted in Figure 1. The hydration of these conformers was performed in a systematic manner adding one explicit molecule of water each time until saturation was reached and also embedding these complexes in a continuum dielectric constant of water, through the PCM model, which allowed us to have a stable zwitterion conformer. The sequence for incorporating the water molecules explicitly was referred to in Figure 2 and Table 1.

The zwitterion structure needs a minimum of two water molecules in the supermolecule structure to preserve its stability in gas phase, but within the PCM approximation the three structures are stable by themselves, i.e. without any explicit water molecule. As a remainder, the SMD variant is a IEF-PCM that includes the non-electrostatic terms for Truhlar and coworkers' solvation model. Within the PCM approximation four water molecules are needed for Z-Gly to become more stable than the whole series of hydrated N-Gly and TS-Gly molecules, see Figure 3.

The energies [in a.u.] for the different super-molecular systems of Gly: $\left(\mathrm{H}_{2} \mathrm{O}\right)_{n}$ (with $\mathrm{n}=1$ to 8$)$ incorporating water molecules one-by-one were obtained as: $E_{G l y}+(8-n)$. $E_{\mathrm{H}_{2} \mathrm{O}}$, where $E_{G l y}$ and $E_{\mathrm{H}_{2} \mathrm{O}}$ are the energies of glycine and water respectively within the PCM model. The obtained energies are represented in Figure 3 together with the energies of each conformer, N, TS and Z, namely with subindex 0 , of 0 water molecule.

Clearly it can be seen two clusters of dots in the structures N-Gly and TS-Gly for $n$ $=1$ and 2 ; and $\mathrm{n}=3$ and 4 , what means that the gaps between $\mathrm{n}=0$ and 1 indicates a decrease in the formation energy due to the formation of a H-bond in the zone labeled AmAc, (1) from Tab. 1. The gap between $\mathrm{n}=2$ and 3 shows the formation of a stronger $\mathrm{H}$-Bond in the zone labeled $O_{T},(3)$ from Tab. 1.

Finally, the gap between $\mathrm{n}=7$ and 8 , that is common for all three structures, again shows a new formation of a stronger $\mathrm{H}$-Bond in the zone labeled $O_{T}$ and the additional H-Bonds formed between water molecules, (8) from Tab. 1. 


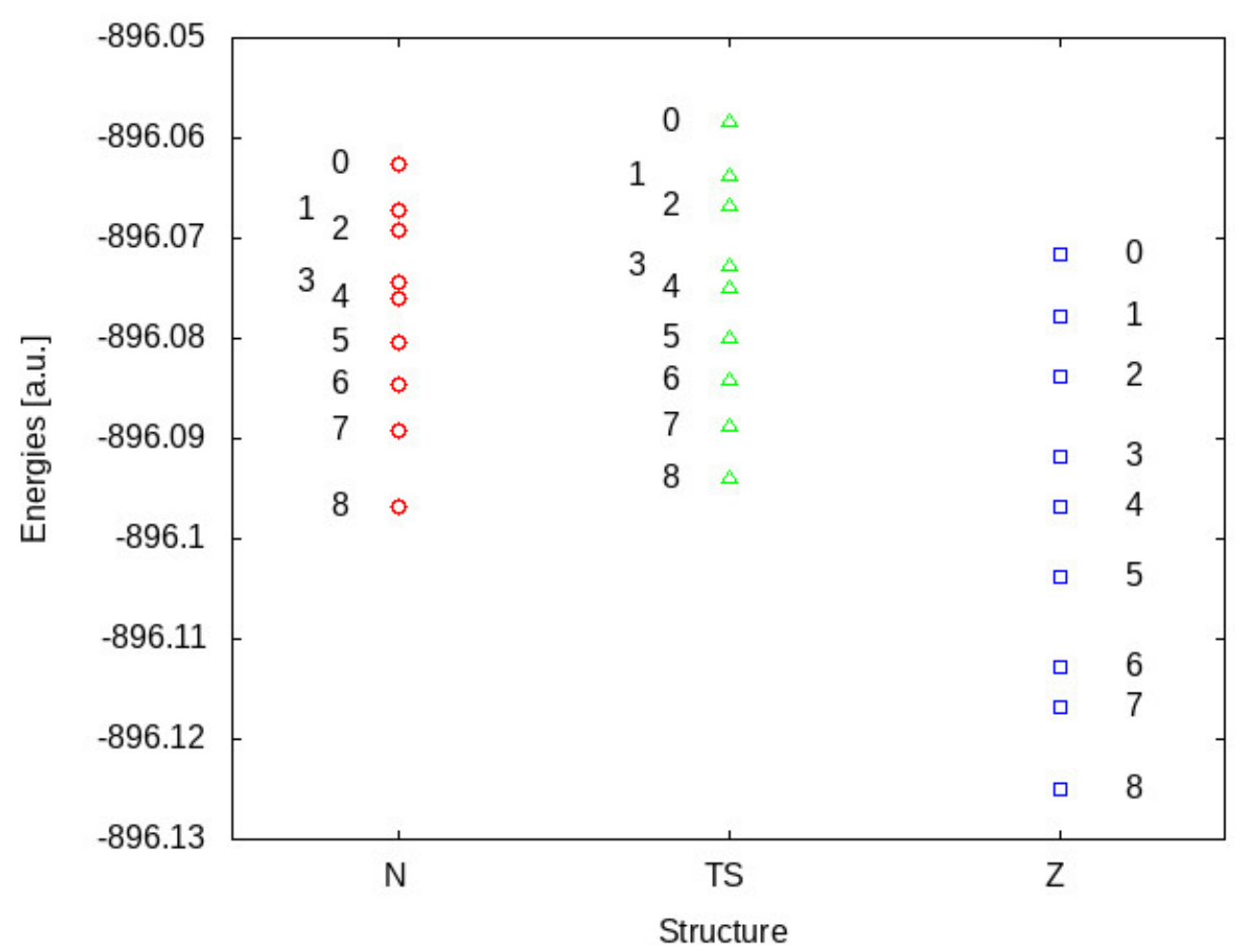

Figure 3. Energies [in a.u.] of the three super-molecular systems Gly: $\left(\mathrm{H}_{2} \mathrm{O}\right)_{n}$ (with $\mathrm{n}=1$ to 8) accounting to the equation: $\left[E_{G l y}+(8-n) \cdot E_{H_{2} O}\right]$

Worth of being mentioned is the fact that addition of water molecule on the Ac zone, (2) from Tab. 1; and in the An zone, (4 and 5) from Tab. 1, have the least effect in the downing of energy in the super-molecule system. While the addition of a second water molecule in the $O_{T}$ zone, (6) from Tab. 1 and in the AmAc zone, (7) from Tab. 1 produce an intermediate downing in the energy of the super-molecule system.

Structure Z-Gly has a slightly different pattern with at least five gaps in its pattern. The first two and the last are equivalent to those just seen for N-Gly and TS-Gly structures. Meanwhile, there are two extra gaps which are between $\mathrm{n}=4$ and 5 , that appear with H-Bond formation between water molecules in the Am zone, (5) from Tab. 1; and the gap between $\mathrm{n}=5$ and 6 , that appears with the formation of the H-bond between water molecules in the $O_{T}$ zone, (6) from Tab. 1. Thus, taking into account the size of the gaps one sees that only the first water molecule added in the Ac and the first water molecule added in the Am zone contribute very little in the downing of the super-molecular system of Z-Gly. All the others produce a stronger downing in the super-molecule energy.

Figure 3 also shows that the super-molecular structures of the Z-Gly: $\mathrm{W}_{n}$ have always lower energies than the other two. From n=4 the Z-Gly structures are under the smallest energies for structures N-Gly and TS-Gly. The super-molecule structures with more than eight water molecules mainly add H-bonds between water molecules than with glycine. We must also mention that the $\mathrm{N}$-Gly can transform into Z-Gly from $\mathrm{n}=2$ on.

The Figure 4 represents the energy difference [in a.u.] between neutral and zwitterionic conformers and their hydrated structures. In all cases the difference of energy, $\Delta E_{N-Z}=E_{N}-E_{Z}$, is positive which agrees well with the pattern observed in Figure 3, where the energies of zwitterion are lower than their neutral counterparts. Only for the super-molecule $\mathrm{n}=7$, Gly:7W, the energy difference, 0.0282 a.u., is slightly smaller than the one for $\mathrm{n}=6,0.0277$ a.u. This fact, together with the almost imperceptible increase for $\mathrm{n}=8,0.0283$ a.u. is directly related to the saturation of the super-molecule systems and the difference between $\mathrm{N}$ and $\mathrm{Z}$ tend to be constant. 


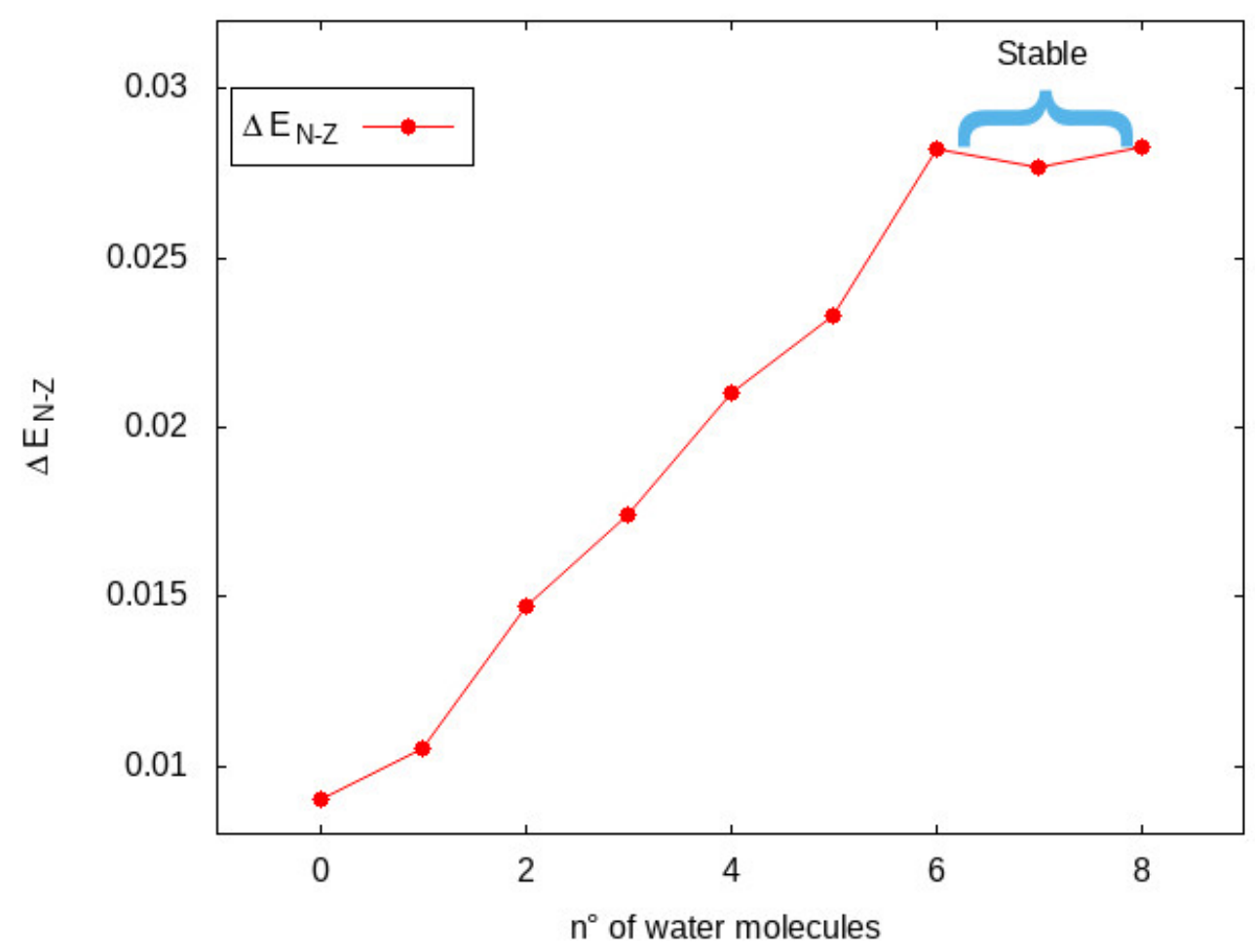

Figure 4. Difference between the energies [in a.u.] of the Glycine, Neutral and Zwitterion conformers, in function of the number of water molecules.

\subsection{Spin-spin Coupling Constants}

The intramolecular SSCC's between the heavy atoms, other than hydrogen, from one to three bonds in glycine have been investigated for the neutral and zwitterionic forms in a electrostatic embedding within PCM and adding to the calculations up to 8 molecules of water in explicit form.

The isotropic values and their contributions are collected in Figures 5 (a) - (c) and Tables ?? -?? of the supplementary material and summarized in Table 2 and 3. 
Table 2. One- two- and three-bond of the SSCC's for N-Gly: $8 \mathrm{~W}$ and Z-Gly: $8 \mathrm{~W}$ (Last column of Tables ?? - ??) of the supplementary material.

\begin{tabular}{clrr|ccc}
\hline Coupling & Molecule & \multicolumn{1}{c}{$0 \mathrm{~W}$} & \multicolumn{1}{c}{$8 \mathrm{~W}$} & PCM $^{a}$ & ASEC-FEG $^{a}$ & Exp. \\
\hline${ }^{1} J\left(C_{O^{\prime} s}, C_{N}\right)$ & $\mathrm{N}$ & 56.3817 & 55.6072 & & & \\
& $\mathrm{Z}$ & 55.0435 & 57.8703 & 42.3 & 56.5 & $53.6{ }^{b, c}$ \\
${ }^{1} J\left(N, C_{N}\right)$ & $\mathrm{N}$ & -5.2400 & -4.8205 & & & \\
& $\mathrm{Z}$ & -4.0884 & -5.4008 & 0.5 & 3.3 & $6.2^{d}$ \\
${ }^{1} J\left(O_{A c}, C_{O^{\prime} s}\right)$ & $\mathrm{N}$ & 29.3819 & 28.5249 & & & \\
& $\mathrm{Z}$ & 35.1057 & 34.5137 & 36.6 & 30.7 & \\
${ }^{1} J\left(O_{T}, C_{O^{\prime} s}\right)$ & $\mathrm{N}$ & 28.5345 & 27.0972 & & & \\
& $\mathrm{Z}$ & 33.6927 & 32.4339 & 37.6 & 31.4 & \\
\hline${ }^{2} J\left(N, C_{O^{\prime} s}\right)$ & $\mathrm{N}$ & -4.9415 & -3.7056 & & & \\
& $\mathrm{Z}$ & 0.0633 & -0.0463 & & & \\
${ }^{2} J\left(O_{A c}, C_{n}\right)$ & $\mathrm{N}$ & -1.8504 & -1.6828 & & & \\
& $\mathrm{Z}$ & -6.9430 & -6.6946 & & & \\
${ }^{2} J\left(O_{T}, C_{N}\right)$ & $\mathrm{N}$ & -3.3170 & -2.7073 & & & \\
& $\mathrm{Z}$ & -10.8332 & -9.2203 & & & \\
${ }^{2} J\left(O_{T}, O_{A c}\right)$ & $\mathrm{N}$ & -0.5083 & -0.1739 & & & \\
& $\mathrm{Z}$ & -4.5047 & -4.9850 & & & \\
\hline${ }^{3} J\left(O_{A c}, N\right)$ & $\mathrm{N}$ & 2.2345 & 1.4947 & & & \\
& $\mathrm{Z}$ & 0.6123 & 0.0539 & & & \\
${ }^{3} J\left(O_{T}, N\right)$ & $\mathrm{N}$ & 0.3740 & 0.2543 & & & \\
& $\mathrm{Z}$ & 0.2178 & 0.1965 & & & \\
\hline
\end{tabular}

The values were respectivelly taken from: ${ }^{a}$ Ref. [19], ${ }^{b}$ Ref. [52], ${ }^{c}$ taken from Ref. [53] and ${ }^{d}$ taken from Ref. [54]

The N- and Z-Gly with just the PCM embedding, 0W, and up to eight explicit water molecules, $8 \mathrm{~W}$, are shown in Table 2 and compared with the best calculations of Valverde et al [19] and same experiments Ref. [52-54]. At first glance can be noted two main characteristics, one is the better agreement with experiment for ${ }^{1} J\left(N, C_{N}\right)$ in the present work and that the values for ${ }^{1} J\left(O_{A c}, C_{O^{\prime} s}\right)$ and ${ }^{1} J\left(O_{T}, C_{O^{\prime} s}\right)$ seems to be exchanged between this work and the work by Valverde et al [19]. Discarding the possibility of human error, according to private communication, then it could arise from differences in the geometries or basis sets, but a further investigation would be needed. 


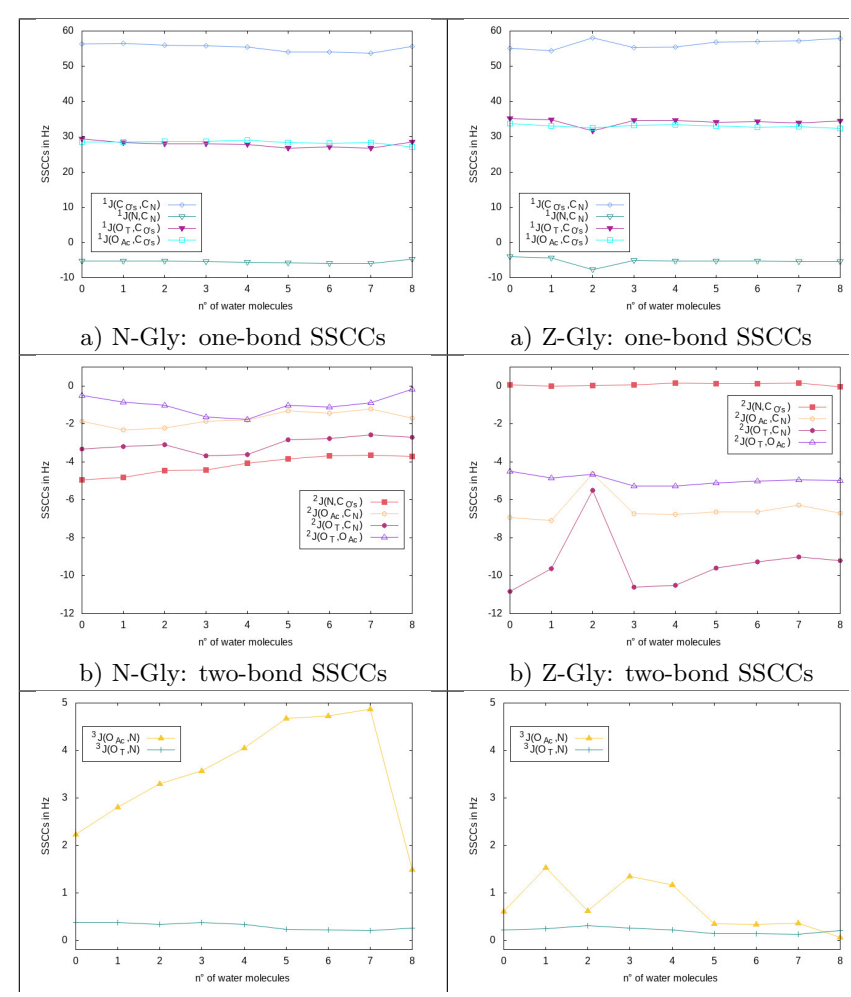

Figure 5. One- (a) two- (b) and three-bond (c) spin-spin coupling constants [in Hz] obtained at the B3LYP/6-311++ $\mathrm{G}^{* *}-\mathrm{J}$ within PCM level of calculations in function of the number of water molecules explicitly incorporated to the calculations.

The complexity of the variations along the series are clearly exposed in the Figure 5 , where can be seen the qualitative general behavior of the intramolecular coupling in glycine. A summarized pattern can be extracted from Table 3. Where are exposed the differences between Gly:4W - Gly:0W, Gly:8W - Gly:4W and the the total difference Gly:8W - Gly:0W which is just the addition of the former two. Also the FC contributions which determine, in general, the behavior of the total are shown.

The one-bond SSCC's change as much as $\sim 3.0 \mathrm{~Hz}$ for Z-Gly, along the series Gly:nW, when incorporating explicitly water molecules, from $\mathrm{n}=0$ to 8 , in the calculations. In particular the ${ }^{1} J\left(C_{O^{\prime} s}, C_{N}\right)$ and ${ }^{1} J\left(N, C_{N}\right)$ of the Z-Gly, $2.83 \mathrm{~Hz}$ and $-1.31 \mathrm{~Hz}$ are the most sensitive to the presence of explicit water than the corresponding counterparts of N-Gly, $-0.77 \mathrm{~Hz}$ and $0.42 \mathrm{~Hz}$, see Fig. 5 (a). While for Z-Gly ${ }^{1} J\left(C_{O^{\prime} s}, C_{N}\right)$ increases with the incorporation of water molecules ${ }^{1} J\left(N, C_{N}\right)$ decreases, and the opposite happens with N-Gly.

The changes in ${ }^{1} J\left(C_{O^{\prime} s}, C_{N}\right)$ for N-Gly arise from the first round of added water molecules and is reduced a bit by the second round. While for Z-Gly the main variation arise from the second round of water molecules and is enlarged a bit by the first round. The changes in ${ }^{1} J\left(N, C_{N}\right)$ for N- and Z-Gly seems to mirror what occurs in ${ }^{1} J\left(C_{O^{\prime} s}, C_{N}\right)$.

The other two one-bond SSCC's, ${ }^{1} J\left(O_{A c}, C_{O^{\prime} s}\right)$ and ${ }^{1} J\left(O_{T}, C_{O^{\prime} s}\right)$, vary much less with the incorporation of explicit water molecules, and for Z-Gly they decrease, $-0.59 \mathrm{~Hz}$ and $-1.26 \mathrm{~Hz}$ respectively. While for N-Gly the decrease is of $-0.8570 \mathrm{~Hz}$ and $-1.4373 \mathrm{~Hz}$.

For N-Gly the changes observed in ${ }^{1} J\left(O_{A c}, C_{O^{\prime} s}\right)$ arise from the first round of added water molecules and is counteracted by the second round in about $46 \%$; while for Z-Gly the second round act in the same direction as the main variation introduced by the first round of added water molecules. Again, in ${ }^{1} J\left(O_{T}, C_{O^{\prime} s}\right)$, the effect of the first and second round of added water molecules for both $\mathrm{N}$ - and Z-Gly seems to mirror the previous behavior. 
Remarkable is the fact that these last two SSCC's have, along the series, an almost constant difference between N-Gly and Z-Gly of about $6.0 \mathrm{~Hz}$ in favor of Z-Gly for the former coupling constant and about $5.0 \mathrm{~Hz}$ in favor of Z-Gly for the latter coupling constant. This characteristic makes any of the one-bond oxygen-carbon SSCC's and excellent marker for distinguishing between the conformers.

The one-bond carbon-carbon and nitrogen-carbon SSCC's contrast with the previous remark, since they do not seems to show much differences between conformers, 5 (a). Instead, they vary in different directions according to the conformer and the coupling constant, see Table 2.

Table 3. Difference of the intramolecular one- two- and three-bond spin-spin coupling constants (in $\mathrm{Hz}$ ) shown in Tables ?? - ?? of the supplementary material, for the three situation: glycine alone $(0 \mathrm{~W})$, glycine +4 water molecules $(4 \mathrm{~W})$ and glycine +8 water molecules $(8 \mathrm{~W})$.

\begin{tabular}{clrrrr|rr}
\hline \multirow{2}{*}{ Coupling } & Molecule & \multicolumn{2}{c}{$4 \mathrm{~W}-0 \mathrm{~W}$} & \multicolumn{2}{c|}{$8 \mathrm{~W}-4 \mathrm{~W}$} & \multicolumn{2}{c}{$8 \mathrm{~W}-0 \mathrm{~W}$} \\
& & $\mathrm{FC}$ & Total & $\mathrm{FC}$ & Total & \multicolumn{1}{c}{$\mathrm{FC}$} & Total \\
\hline${ }^{1} J\left(C_{O^{\prime} s}, C_{N}\right)$ & $\mathrm{N}$ & -0.91 & -0.94 & 0.38 & 0.17 & -0.53 & -0.77 \\
& $\mathrm{Z}$ & 0.60 & 0.47 & 2.53 & 2.36 & 3.13 & 2.83 \\
${ }^{1} J\left(N, C_{N}\right)$ & $\mathrm{N}$ & -0.45 & -0.35 & 0.77 & 0.77 & 0.31 & 0.42 \\
& $\mathrm{Z}$ & -1.17 & -1.23 & -0.15 & -0.09 & -1.32 & -1.31 \\
${ }^{1} J\left(O_{A c}, C_{O^{\prime} s}\right)$ & $\mathrm{N}$ & -1.71 & -1.60 & 1.05 & 0.74 & -0.66 & -0.86 \\
& $\mathrm{Z}$ & -0.28 & -0.41 & -0.14 & -0.18 & -0.42 & -0.59 \\
${ }^{1} J\left(O_{T}, C_{O^{\prime} s}\right)$ & $\mathrm{N}$ & 0.35 & 0.43 & -1.81 & -1.87 & -1.46 & -1.44 \\
& $\mathrm{Z}$ & -0.32 & -0.23 & -1.35 & -1.02 & -1.67 & -1.26 \\
\hline${ }^{2} J\left(N, C_{O^{\prime} s}\right)$ & $\mathrm{N}$ & 0.86 & 0.87 & 0.38 & 0.37 & 1.24 & 1.24 \\
& $\mathrm{Z}$ & 0.11 & 0.10 & -0.22 & -0.21 & -0.11 & -0.11 \\
${ }^{2} J\left(O_{A c}, C_{N}\right)$ & $\mathrm{N}$ & 0.03 & 0.06 & 0.12 & 0.11 & 0.15 & 0.17 \\
& $\mathrm{Z}$ & 0.09 & 0.18 & 0.03 & 0.07 & 0.12 & 0.25 \\
${ }^{2} J\left(O_{T}, C_{N}\right)$ & $\mathrm{N}$ & -0.29 & -0.31 & 0.89 & 0.92 & 0.61 & 0.61 \\
& $\mathrm{Z}$ & 0.23 & 0.34 & 1.25 & 1.27 & 1.48 & 1.61 \\
${ }^{2} J\left(O_{T}, O_{A c}\right)$ & $\mathrm{N}$ & -1.36 & -1.25 & 1.44 & 1.58 & 0.08 & 0.33 \\
& $\mathrm{Z}$ & -0.83 & -0.78 & 0.44 & 0.30 & -0.40 & -0.48 \\
\hline${ }^{3} J\left(O_{A c}, N\right)$ & $\mathrm{N}$ & 1.89 & 1.82 & -2.69 & -2.56 & -0.80 & -0.74 \\
& $\mathrm{Z}$ & 0.59 & 0.56 & -1.21 & -1.12 & -0.63 & -0.56 \\
${ }^{3} J\left(O_{T}, N\right)$ & $\mathrm{N}$ & -0.06 & -0.04 & -0.05 & -0.08 & -0.10 & -0.12 \\
& $\mathrm{Z}$ & -0.03 & 0.00 & 0.03 & -0.02 & 0.00 & -0.02 \\
\hline
\end{tabular}

It is worth to note that due to the difference in scale of ordinate axes between Figures 5 (a), (b) and (c), the variations observed for two- and three-bonds SSCC's are not that much as they look like at first glance, instead they all are rather quite stable.

The general behavior of all gemial SSCC's is that they are roughly stable between $\mathrm{n}=0$ and 8 , with the addition of explicit water molecules with only two exceptions, ${ }^{2} J\left(N, C_{O^{\prime} s}\right)$ and ${ }^{2} J\left(O_{T}, C_{N}\right)$ which shows changes over $1.0 \mathrm{~Hz}$ for N-Gly and Z-Gly respectively, see Table 3 .

The Figure 5 (b) also let one sees that ${ }^{2} J\left(N, C_{O^{\prime} s}\right)$ has a steady increment, along the series, for N-Gly but it is almost unchanged for Z-Gly, just $0.1 \mathrm{~Hz}$. In the same manner ${ }^{2} J\left(O_{T}, O_{A c}\right)$ decreases steadily for the the first round of water molecules and increase a bit for the second round producing a net change of $-0.5 \mathrm{~Hz}$ for Z-Gly and stays standing for N-Gly. However, the average difference along the series between conformers is roughly constant and about $4.0 \mathrm{~Hz}$ in both cases. Again, this fact would let experiments distinguish between conformers in a quite reliable manner. 
For the two-bond SSCC's the second round of added water molecules are more influential than the first round, with only two exceptions, ${ }^{2} J\left(O_{A c}, C_{N}\right)$ and ${ }^{2} J\left(O_{T}, O_{A c}\right)$, in both cases for Z-Gly.

The net changes from $0 \mathrm{~W}$ to $8 \mathrm{~W}$ in the three-bond SSCC's are between the smallest and are of only a few tenth, Table 3. However, Figure 5 (c) shows large variations in the intermediate stages of ${ }^{3} J\left(O_{A c}, N\right)$ for both compounds, but mainly for N-Gly. These large variations can be attributable to the geometrical changes in the conformation of hydrogens in the glycines compounds, $\mathrm{N}$ and $\mathrm{Z}$, which affect more to long nuclear interactions, mainly due to their small values.

The ${ }^{3} J\left(O_{T}, N\right)$ coupling constants shows similar trends and magnitudes for both compounds. This fact make such coupling constant very reliable for experiments but would be very difficult to distinguish between conformers.

As seen previously, at least two of the four stable SSCC's that show a large difference between $\mathrm{N}$ - and Z-Gly contain at least one oxygen, ${ }^{1} J\left(N, C_{N}\right),{ }^{1} J\left(O_{A c}, C_{O^{\prime} s}\right)$, ${ }^{2} J\left(N, C_{O^{\prime} s}\right)$ and ${ }^{2} J\left(O_{T}, O_{A c}\right)$, see Fig. 5 and Tab. ?? - ?? of the supplementary material.

The ${ }^{3} J\left(O_{T}, N\right)$ SSCC is very stable but shows similar magnitudes for both conformers therefore is unable to discern between them.

Thus, it is worth to mention that the oxygen, in general, has received less attention than the other elements of the first and second row of the periodic table, mainly due to the small abundance of its only magnetic nucleus, which is $0.037 \%$ and the fact that it possess a nuclear spin $\mathrm{I}=5 / 2$ that implies a quadrupole moments, responsible of the broadening of the NMR line widths, see for instance Ref. [55-62] among many others.

Moreover, it is also worth to note that for the geminal coupling constants ${ }^{2} J\left(O_{T}, O_{A c}\right)$ the PSO contribution is dominant by far and determine the sign of the coupling constant. The FC contribution is only a $75 \%$ of it. Hardly the FC and DSO contributions compensate the dominance of PSO contribution. For the ${ }^{1} J\left(O_{A c}, C_{O^{\prime} s}\right),{ }^{1} J\left(O_{T}, C_{O^{\prime} s}\right)$ coupling constants the PSO contributions are about $30 \%$ of the total.

\section{Conclusions}

In this work we have studied systematically the energy and the intramolecular SSCC's of the heavy nuclei, other than hydrogens, for neutral and zwitterionic glycine within a dielectric constant of water using the PCM model and including explicit water molecules increasing the number of them from zero to eight where all geometrical parameters are allowed to relax.

Within the PCM embedding the three structures, N, Z and TS, are stable. The zwitterionic structure needs at least two explicit water molecules in the super- molecule structure to preserve its stability in gas phase and four explicit water molecule to become more stable than the whole series of hydrated neutral molecule.

From six to eight water molecules the energy difference between N-Gly and Z-Gly becomes approximately constant.

The analyses of the intramolecular SSCC's reflexes, along the series, some very stable coupling constants for both conformers, like the four one-bond coupling constants, ${ }^{1} J\left(C_{O^{\prime} s}, C_{N}\right),{ }^{1} J\left(N, C_{N}\right),{ }^{1} J\left(O_{A c}, C_{O^{\prime} s}\right)$ and ${ }^{1} J\left(O_{T}, C_{O^{\prime} s}\right)$; two of the two-bond SSCC's, ${ }^{2} J\left(N, C_{O^{\prime} s}\right)$ and ${ }^{2} J\left(O_{T}, O_{A c}\right)$; and one of the three-bond SSCC's, ${ }^{3} J\left(O_{T}, N\right)$. Three of these coupling constants have very similar magnitude to each other conformer, i.e. they do not distinguish either between conformer nor accuse changes in the environment. This is the case of SSCC's ${ }^{1} J\left(C_{O^{\prime} s}, C_{N}\right),{ }^{1} J\left(N, C_{N}\right)$ and ${ }^{3} J\left(O_{T}, N\right)$.

Therefore the other four SSCC's are also very stable along the series, they almost do not accuse changes in the environment, but they exhibit quite different magnitudes for both conformers that is over $4.0 \mathrm{~Hz}$, making them excellent markers for experiments. 
Acknowledgments: The author acknowledge financial support to the present research from CONICET (PIP 112230100377).

\section{References}

1. Jensen, J.H.; Gordon, M.S. On the Number of Water Molecules Necessary To Stabilize the Glycine Zwitterion. J. Am. Chem. Soc. 1995, 117, 8159-8170.

2. Fernandez-Ramos, A.; Smedarchina, Z.; Siebrand, W.; Zgierski, M.Z. A direct-dynamics study of the zwitterion-to-neutral interconversion of glycine in aqueous solution. J. Chem. Phys. 2000, 113, 9714-9721.

3. Karmacharya, R.; Antoniou, D.; Schwartz, S.D. Nonequilibrium Solvation and the Quantum Kramers Problem: Proton Transfer inAqueous Glycine. J. Phys. Chem. A 2001, 105, 25632567.

4. Aikens, C.M.; Gordon, M.S. Incremental Solvation of Nonionized and Zwitterionic Glycine. J. Am. Chem. Soc. 2006, 128, 12835-12850.

5. Leung, K.; Rempe, S.B. Ab initio molecular dynamics study of glycine intramolecular proton transfer in water. J. Chem. Phys. 2005, 122, 184506(1-13).

6. Campo, M.G. Molecular dynamics simulation of glycine zwitterion in aqueous solution. $J$. Chem. Phys. 2006, 125, 114511(1-9).

7. Balabin, R.M. The First Step in Glycine Solvation: The Glycine-Water Complex. J. Phys. Chem. B 2010, 114, 15075-15078.

8. Takenaka, N.; Kitamura, Y.; Koyano, Y.; Asada, T.; Nagaoka, M. Reaction path optimization and vibrational frequency analysis via ab initio QM/MM free energy gradient (FEG) method: application to isomerization process of glycine in aqueous solution. Theor. Chem. Acc 2011, 130, 215-226.

9. Kim, J.Y.; Ahn, D.S.; Park, S.W.; Lee, S. Gas phase hydration of amino acids and dipeptides: effects on the relative stability of zwitterion vs. canonical conformers. $R S C$ Adv. 2014, 4, 16352-16361.

10. Wada, G.; Tamura, E.; Okina, M.; Nacamura, M. On the ratio of zuitterion form to uncharged form of glycine at equilibrium in various aqueous media. Bull. Chem. Soc. Jpn. 1982, 55, 3064-3067.

11. Slifkin, M.A.; All, S.M. Thermodinamic parameters of the activation of glycine zwitterion protonation reactions. J. Mol. Liq. 1984, 28, 215-221.

12. Peteanu, L.A.; Levy, D.H. Spectroscopy of Complexes of Tryptamine and 3-Indolepropionic Acid with Various Solvents. J . Phys. Chem. 1988, 92, 6554-6561.

13. Xu, S.; Nilles, J.M.; Bowen, K.H. Zwitterion formation in hydrated amino acid, dipole bound anions: How many water molecules are required? J. Chem. Phys. 2003, 119, 10696(1-7).

14. Diken, E.G.; Hammer, N.I.; Johnson, M.A. Preparation and photoelectron spectrum of the glycine molecular anion: Assignment to a dipole-bound electron species with a high-dipole moment, non-zwitterionic form of the neutral core. J. Chem. Phys. 2004, 120, 9899-9902.

15. Nonose, S.; Iwaoka, S.; Mori, K.; Shibata, Y.; Fuke, K. Structures and reactions of hydrated biomolecular cluster ions. Eur. Phys. J. D 2005, 34, 315-319.

16. Alonso, J.L.; Cocinero, E.J.; Lesarri, A.; Sanz, M.E.; López, J.C. The Glycine-Water Complex. Angew. Chem. 2006, 118, 3551-3554.

17. da Silva, A.M.; Ghosh, A.; Chaudhuri, P. Effect of Hydrogen Bond Formation on the NMR Properties of Glycine-HCN Complexes. J. Phys. Chem. A 2013, 117, 10274-10285.

18. Chaudhuri, Puspitapallab; Canuto, S.P.P.F. NMR spin-spin coupling constants in hydrogenbonded glycine clusters. International Journal of Quantum Chemistry 2018. doi: 10.1002/qua.25608.

19. Valverde, D.; da Costa Ludwig, Z.M.; da Costa, C.R.; Ludwig, V.; Georg, H.C. Zwitterionization of glycine in water environment: Stabilization mechanism and NMR spectral signatures. The Journal of Chemical Physics 2018, 148, 024305,(1-8). doi:10.1063/1.5006645.

20. Arroyuelo, A.; Martin, O.A.; Scheraga, H.A.; Vila, J.A. Assessing the One-Bond C $\alpha-\mathrm{H}$ Spin-Spin Coupling Constants in Proteins: Pros and Cons of Different Approaches. J. Phys. Chem. B 2020, 124, 735-741.

21. Császár, A.G. Conformers of Gaseous Glycine. J. Am. Chem. Soc. 1992, 114, 9568-9575.

22. Godfrey, P.D.; Brown, R.D.; Rodgers, F.M. The missing conformers of glycine and alanine: relaxation in seeded supersonic jets'. J. Mol. Struct. 1996, 376, 65-81. 
23. Sauer, S.P.A.; Oddershede, J.; Sabin, J.R. Directional Dependence of the Mean Excitation Energy and Spectral Moments of the Dipole Oscillator Strength Distribution of Glycine and Its Zwitterion. J. Phys. Chem. A 2006, 110, 8811-8817.

24. Caputo, M.C.; Provasi, P.F.; Sauer, S.P.A. The role of explicit solvent molecules in the calculation of NMR chemical shifts of glycine in water. Theor. Chem. Acc. 2018, 137, 88 $(1-8)$.

25. Ding, Y.; Krogb-Jespersen, K. The glycine zwitterion does not exist in the gas phase: results from a detailed ab initio electronic structure study. Chem. Phys. Lett. 1992, 199, 261-266.

26. Frisch, M.J.; Trucks, G.W.; Schlegel, H.B.; Scuseria, G.E.; Robb, M.A.; Cheeseman, J.R.; Scalmani, G.; Barone, V.; Mennucci, B.; Petersson, G.A.; Nakatsuji, H.; Caricato, M.; Li, X.; Hratchian, H.P.; Izmaylov, A.F.; Bloino, J.; Zheng, G.; Sonnenberg, J.L.; Hada, M.; Ehara, M.; Toyota, K.; Fukuda, R.; Hasegawa, J.; Ishida, M.; Nakajima, T.; Honda, Y.; Kitao, O.; Nakai, H.; Vreven, T.; Jr., J.A.M.; Peralta, J.E.; Ogliaro, F.; Bearpark, M.; Heyd, J.J.; Brothers, E.; Kudin, K.N.; Staroverov, V.N.; Kobayashi, R.; Normand, J.; Raghavachari, K.; Rendell, A.; Burant, J.C.; Iyengar, S.S.; Tomasi, J.; Cossi, M.; Rega, N.; Millam, J.M.; Klene, M.; Knox, J.E.; Cross, J.B.; Bakken, V.; Adamo, C.; Jaramillo, J.; Gomperts, R.; Stratmann, R.E.; Yazyev, O.; Austin, A.J.; Cammi, R.; Pomelli, C.; Ochterski, J.W.; Martin, R.L.; Morokuma, K.; Zakrzewski, V.G.; Voth, G.A.; Salvador, P.; Dannenberg, J.J.; Dapprich, S.; Daniels, A.D.; Ö. Farkas.; Foresman, J.B.; Ortiz, J.V.; Cioslowski, J.; Fox, D.J. Gaussian 09 Revision D.01; Gaussian Inc. Wallingford CT, 2009.

27. Becke, A.D. Density-functional thermochemistry. III. The role of exact exchange. J. Chem. Phys. 1993, 98, 5648-5652.

28. Lee, C.; Yang, W.; Parr, R.G. Development of the Colle-Salvetti correlation-energy formula into a functional of the electron density. Phys. Rev. B 1988, 37, 785-789.

29. Clark, T.; Chandrasekhar, J.; Spitznagel, G.W.; Schleyer, P.V.R. Efficient diffuse functionaugmented basis sets for anion calculations. III. The $3-21+\mathrm{G}$ basis set for first-row elements, Li-F. J. Comput. Chem., 4, 294-301 (1983) 1983, 4, 294-301.

30. Ditchfield, R.; Hehre, W.J.; Pople, J.A. Self-Consistent Molecular-Orbital Methods. IX. An Extended Gaussian- Type Basis for Molecular-Orbital Studies of Organic Molecules. J. Chem. Phys. 1971, 54, 724-728.

31. Hariharan, P.C.; Pople, J.A. The influence of polarization functions on molecular orbital hydrogenation energies. Theor. Chim. Acta 1973, 28, 213-222.

32. Hehre, W.J.; Ditchfield, R.; Pople, J.A. Self-Consistent Molecular Orbital Methods. XII. Further Extensions of Gaussian-Type Basis Sets for Use in Molecular Orbital Studies of Organic Molecules. J. Chem. Phys. 1972, 56, 2257-2261.

33. Kjær, H.; Sauer, S.P.A. Pople Style Basis Sets for the Calculation of NMR Spin-Spin Coupling Constants: the 6-31G-J and 6-311G-J Basis Sets. J. Chem. Theory Comput. 2011, 7, 4070-4076.

34. Pritchard, B.P.; Altarawy, D.; Didier, B.; Gibsom, T.D.; Windus, T.L. A New Basis Set Exchange: An Open, Up-to-date Resource for the Molecular Sciences Community. J. Chem. Inf. Model. 2019, 59, 4814-4820.

35. Feller, D. The role of databases in support of computational chemistry calculations. J. Comput. Chem. 1996, 17, 1571-1586. doi:10.1002/(SICI)1096-987X(199610)17:13<1571::AIDJCC9>3.0.CO;2-P.

36. Schuchardt, K.L.; Didier, B.T.; Elsethagen, T.; Lisong Sun, L.; Gurumoorthi, V.; Chase, J.; Li, J.; Windus, T.L. Basis Set Exchange: A Community Database for Computational Sciences. Magn. Reson. Chem. 2007, 47, 1045-1052.

37. Mennucci, B.; Tomasi, J. Continuum solvation models: A new approach to the problem of solute's charge distribution and cavity boundaries. J. Chem. Phys. 1997, 106, 5151-5158.

38. Marenich, A.V.; Cramer, C.J.; Truhlar, D.G. Universal Solvation Model Based on Solute Electron Density and on a Continuum Model of the Solvent Defined by the Bulk Dielectric Constant and Atomic Surface Tensions. J. Phys. Chem. B 2009, 113, 6378-6396.

39. Miertus, S.; Scroco, E.; Tomasi, J. Electrostatic interaction of a solute with a continuum. A direct utilizaion of ab initio molecular potentials for the prevision of solvent effects. Chem. Phys. 1981, 55, 117-129.

40. Miertus, S.; Tomasi, J. Approximate evaluations of the electrostatic free energy and internal energy changes in solution processes. Chem. Phys. 1982, 65, 239-245. 
41. Tomasi, J.; Mennucci, B.; Cammi, R. Quantum Mechanical Continuum Solvation Models. Chem. Rev. 2005, 105, 2999-3093.

42. Cances, E.; Mennucci, B.; Tomasi, J. A new integral equation formalism for the polarizable continuum model: Theoretical background and applications to isotropic and anisotropic dielectrics. J. Chem. Phys. 1997, 10\%, 3032.

43. Cances, E.; Mennucci, B. New applications of integral equations methods for solvation continuum models: ionic solutions and liquid crystals. J. Math. Chem. 1998, 23, 309-326.

44. Ramsey, N.F. Electron Coupled Interactions between Nuclear Spins in Molecules. Phys. Rev. 1953, 91, 303-307.

45. Autschbach, J.; Guennic, B.L. Analyzing and interpreting NMR spin-spin coupling constants using molecular orbitalcalculations. J. Chem. Educ. 2007, 84, 156-171.

46. Sauer, S.P.A. Molecular Electromagnetism : A Computational Chemistry Approach; Oxford University Press, Oxford, 2011.

47. Helgaker, T.; Jaszuński, M.; Ruud, K. Ab Initio Methods for the Calculation of NMR Shielding and Indirect Spin-Spin Coupling Constants. Chem. Rev. 1999, 99, 293-352.

48. Krivdin, L.B.; Contreas, R.H. Recent advances in theoretical calculations of indirect spin-spin coupling constants. Annu. Rep. NMRSpectrosc. 2007, 61, 133-245.

49. Vaara, J. Theory and computation of nuclear magnetic resonance parameters. Phys. Chem. Chem. Phys. 2007, 9, 5399-5418.

50. Helgaker, T.; Jaszunski, M.; Pecul, M. The quantum-chemical calculation of NMR indirect spin-spin coupling constants. Prog. Nucl. Magn. Reson. Spectrosc. 2008, 53, 249-268.

51. Helgaker, T.; Coriani, S.; Jørgensen, P.; Kristensen, K.; Olsen, J.; Ruud, K. Recent advances in wave function-based methods of molecular-property calculations. Chem. Rev. 2012, 112, 543-631.

52. Sogn, J.A.; Craig, L.C.; Gibbons, W.A. Carbon-13-carbon-13 coupling constants in a series of carbon-13-enriched amino acids. J. Am.Chem. Soc. 1974, 96, 4694-4696.

53. Fermandjian, S.; Trandinh, S.; Savrda, J.; Sala, E.; Mermetbouvier, R.; Bricas, E.; Fromageot, P. ${ }^{13} \mathrm{C}$-nuclear magnetic resonance studies of $85 \%{ }^{13} \mathrm{C}$-enriched amino acids and small peptides: $\mathrm{pH}$ effects on the chemical shifts, coupling constants, kinetics of cis-trans isomerisation and conformation aspects. Biochim. Biophys. Acta, Gen. Subj. 1975, 399, 313-338.

54. Lichter, R.L.; Roberts, J.D. ${ }^{15}$ N Nuclear Magnetic Resonance Spectroscopy. XIII. Pyridine${ }^{15} \mathrm{~N}^{1}$. J. Am. Chem. Soc. 1971, 93, 5218-5224.

55. Lemaitre, V.; Smith, M.E.; Watts., A. A review of oxygen-17 solid-state NMR of organic materials-towards biological applications. Solid State Nucl. Magn. Reson. 2004, 26, 215235.

56. Sergeyev, N.M.; Sergeyeva, N.D.; Strelenko, Y.A.; Raynes, W.T. The ${ }^{1} \mathrm{H}_{-}{ }^{2} \mathrm{H},{ }^{17} \mathrm{O}-{ }^{1} \mathrm{H}$ coupling constants and the ${ }^{16} \mathrm{O} /{ }^{18} \mathrm{O}$ induced proton isotope shift in water. Chem. Phys. Lett. 1997, 277, 142-146.

57. Halle, B.; Karlstrom, G. Prototropic charge migration in water. Part 1.-Rate constants in light and heavy water and in salt solution from oxygen-17 spin relaxation. J. Chem. Soc., Faraday Trans. 2 1983, 79, 1031-1046.

58. Broze, M.; Luz, Z. Oxygen-17 spin-spincoupling with manganese-55 and carbon-13. J. Phys. Chem. 1969, 73, 1600-1602.

59. Klemperer, W.G. 17O-NMR Spectroscopy as a Structural Probe. Angew. Chem. Int. Ed. Engl. 1978, 17, 246-254.

60. Amour, T.S.; Fiat, D. Oxygen-17 Magnetic Resonance. Bull. Magn. Reson. 1979, 1, 118-129.

61. Gerothanassis, I.P. Oxygen-17 NMR spectroscopy: Basic principles and applications (Part I). Prog. Nucl. Magn. Reson. Spectrosc. 2010, 56, 95-197.

62. Gerothanassis, I.P. Oxygen-17 NMR spectroscopy: Basic principles and applications (part II). Prog. Nucl. Magn. Reson. Spectrosc. 2010, 57, 1-110. 
